\title{
ON SOLVABILITY OF GENERAL NONLINEAR \\ VARIATIONAL-LIKE INEQUALITIES \\ IN REFLEXIVE BANACH SPACES
}

\author{
ZEQING LIU, JUHE SUN, SOO HAK SHIM, AND SHIN MIN KANG
}

Received 28 December 2004

We introduce and study a new class of general nonlinear variational-like inequalities in reflexive Banach spaces. By applying a minimax inequality, we establish two existence and uniqueness theorems of solutions for the general nonlinear variational-like inequality.

\section{Introduction}

Recently, variational inequality theory has been extended and applied in various directions, see $[6,7,8,9,10]$ and the references therein. In particular, Ding $[1,2,3]$, and Ding and Tan [4] studied the existence of solutions for several nonlinear variational-like inequalities in reflexive Banach spaces.

In this paper, a new class of general nonlinear variational-like inequalities in reflexive Banach spaces are introduced. Utilizing a minimax inequality due to Ding and Tan [4], we provide some efficient conditions, which ensure the existence and uniqueness of solutions for the general nonlinear variational-like inequality. Our results improve and generalize many known results in the literature.

\section{Preliminaries}

Let $D$ be a nonempty convex subset of a reflexive Banach space $B$ with dual space $B^{*}$ and let $\langle u, v\rangle$ be the dual pairing between $u \in B^{*}$ and $v \in B$. Let $T, A: D \rightarrow B^{*}, N: B^{*} \times B^{*} \rightarrow$ $B^{*}$, and $\eta: D \times D \rightarrow B$ be mappings. Suppose that $a: B \times B \rightarrow(-\infty,+\infty)$ is a coercive continuous bilinear form, that is, there exist positive constants $c$ and $d$ such that

(c1) $a(u, v) \geq c\|v\|^{2}$ for all $v \in B$;

(c2) $a(u, v) \leq d\|u\|\|v\|$ for all $u, v \in B$.

Clearly, $c \leq d$.

Let $f: D \rightarrow(-\infty,+\infty]$ be a real functional and let $z^{*} \in B^{*}$. We consider the following general nonlinear variational-like inequality problem: find $u \in D$ such that

$$
\left\langle N(T u, A u)-z^{*}, \eta(v, u)\right\rangle+a(u, v-u) \geq f(u)-f(v), \quad \forall v \in D .
$$

We have the following special cases. 
1416 General nonlinear variational-like inequalities

(A) If $N(T u, A u)=T u-A u, a(u, v) \equiv 0$ for all $u, v \in D$, and $z^{*}=0$, then the general nonlinear variational-like inequality (2.1) reduces to

$$
\langle T u-A u, \eta(v, u)\rangle \geq f(u)-f(v), \quad \forall v \in D,
$$

which was introduced and studied by Ding [1].

(B) If $N(T u, A u)=T u-A u$ and $a(u, v) \equiv 0$ for all $u, v \in D$, then the general nonlinear variational-like inequality $(2.1)$ reduces to

$$
\left\langle T u-A u-z^{*}, \eta(v, u)\right\rangle \geq f(u)-f(v), \quad \forall v \in D,
$$

which was studied by Yao [10] in Hilbert spaces.

Definition 2.1. Let $D$ be a nonempty subset of a reflexive Banach space $B$ with dual space $B^{*}$. Let $T: D \rightarrow B^{*}, N: B^{*} \times B^{*} \rightarrow B^{*}$, and $\eta: D \times D \rightarrow B$ be mappings.

(1) $T$ is said to be Lipschitz continuous with constant $\alpha$ if there exists a constant $\alpha>0$ such that

$$
\|T u-T v\| \leq \alpha\|u-v\|, \quad \forall u, v \in D .
$$

(2) $N$ is said to be $\eta$-relaxed monotone with constant $\gamma$ with respect to $T$ in the first argument if there exists a constant $\gamma>0$ such that

$$
\langle N(T u, w)-N(T v, w), \eta(u, v)\rangle \geq-\gamma\|u-v\|^{2}, \quad \forall u, v \in D, w \in B^{*} .
$$

(3) $N$ is said to be $\eta$-strongly monotone with constant $\xi$ with respect to $T$ in the first argument if there exists a constant $\xi>0$ such that

$$
\langle N(T u, w)-N(T v, w), \eta(u, v)\rangle \geq \xi\|u-v\|^{2}, \quad \forall u, v \in D, w \in B^{*} .
$$

(4) $N$ is said to be $\eta$-monotone with respect to $A$ in the second argument if

$$
\langle N(w, A u)-N(w, A v), \eta(u, v)\rangle \geq 0, \quad \forall u, v \in D, w \in B^{*} .
$$

(5) $\eta$ is said to be Lipschitz continuous with constant $\delta$ if there exists a constant $\delta>0$ such that

$$
\|\eta(u, v)\| \leq \delta\|u-v\|, \quad \forall u, v \in D .
$$

(6) $\eta$ is said to be strongly monotone with constant $\tau$ if there exists a constant $\tau>0$ such that

$$
\langle u-v, \eta(u, v)\rangle \geq \tau\|u-v\|^{2}, \quad \forall u, v \in D .
$$

Definition 2.2. Let $D$ be a nonempty convex subset of a reflexive Banach space $B$ and let $f: D \rightarrow(-\infty,+\infty]$ be a real functional.

(1) $f$ is said to be convex if

$$
f(\alpha u+(1-\alpha) v) \leq \alpha f(u)+(1-\alpha) f(v), \quad \forall u, v \in D, \alpha \in[0,1] .
$$


(2) $f$ is said to be lower semicontinuous on $D$ if for each $\alpha \in(-\infty,+\infty$, the set $\{u \in$ $D: f(u) \leq \alpha\}$ is closed in $D$.

Definition 2.3. Let $D$ be a nonempty subset of a reflexive Banach space $B$ with dual space $B^{*}$ and let $T: D \rightarrow B^{*}$ and $\eta: D \times D \rightarrow B$ be two mappings. $T$ and $\eta$ are said to have 0 -diagonally concave relation with respect to $z^{*} \in B^{*}$ if the functional $\varphi: D \times D \rightarrow$ $(-\infty,+\infty]$ defined by $\varphi(u, v)=\left\langle T u-z^{*}, \eta(u, v)\right\rangle$ is 0 -diagonally concave in $v$, that is, for any finite set $\left\{v_{1}, \ldots, v_{m}\right\} \in D$ and for any $u=\sum_{i=1}^{m} \lambda_{i} v_{i}$ with $\lambda_{i} \geq 0$ and $\sum_{i=1}^{m} \lambda_{i}=1$,

$$
\sum_{i=1}^{m} \lambda_{i} \varphi\left(u, v_{i}\right) \leq 0
$$

Remark 2.4. It is easy to see that, if for each $u \in D, \eta(u, u)=0$ and the functional $v \mapsto$ $\left\langle T u-z^{*}, \eta(u, v)\right\rangle$ is concave, then the mappings $T$ and $\eta$ have the 0 -diagonally concave relation with respect to $z^{*}$ on $D$.

Lemma 2.5 [4]. Let $D$ be a nonempty convex subset of a topological vector space and let $\varphi: D \times D \rightarrow[-\infty,+\infty]$ be such that

(a) for each $u \in D, v \mapsto \varphi(u, v)$ is lower semicontinuous on each nonempty compact subset of $D$,

(b) for each nonempty finite set $\left\{v_{1}, \ldots, v_{m}\right\} \in D$ and for any $u=\sum_{i=1}^{m} \lambda_{i} v_{i}$ with $\lambda_{i} \geq 0$ and $\sum_{i=1}^{m} \lambda_{i}=1, \min _{1 \leq i \leq m} \varphi\left(u, v_{i}\right) \leq 0$,

(c) there exist a nonempty compact convex subset $X_{0}$ of $D$ and a nonempty compact subset $K$ of $D$ such that for each $v \in D-K$, there is $u \in \operatorname{co}\left(X_{0} \cup\{v\}\right)$ with $\varphi(u, v)>0$.

Then there exists $\hat{v} \in K$ such that $\varphi(u, \hat{v}) \leq 0$ for all $u \in D$.

\section{Existence and uniqueness theorems}

In this section, we use the minimax inequality technique due to Ding and Tan [4] to prove the existence and uniqueness theorems of solutions for the general nonlinear variationallike inequality (2.1).

Theorem 3.1. Let $D$ be a nonempty closed convex subset of a reflexive Banach space $B$ with dual space $B^{*}$. Assume that $\eta: D \times D \rightarrow B$ is Lipschitz continuous with constant $\delta$, for each $v \in D, \eta(\cdot, v)$ is continuous on $D$, and $\eta(v, u)=-\eta(u, v)$ for all $v, u \in D$. Suppose that $a: B \times B \rightarrow(-\infty,+\infty)$ is a coercive continuous bilinear form and $f: D \rightarrow(-\infty,+\infty]$ is a proper convex lower semicontinuous functional with $\operatorname{int}(\operatorname{dom} f) \cap D \neq \varnothing$. Let $T, A$ : $D \rightarrow B^{*}$ and $N: B^{*} \times B^{*} \rightarrow B^{*}$ be continuous mappings, let $N$ be $\eta$-strongly monotone with constant $\alpha$ with respect to $T$ in the first argument and $\eta$-monotone with respect to $A$ in the second argument. Assume that $N$ and $\eta$ have the 0 -diagonally concave relation with respect to $z^{*} \in B^{*}$. Then the general nonlinear variational-like inequality (2.1) has a unique solution $\hat{u} \in D$.

Proof. Define a functional $\varphi: D \times D \rightarrow(-\infty,+\infty]$ by

$$
\varphi(v, u)=\left\langle z^{*}-N(F u, G u), \eta(v, u)\right\rangle-a(u, v-u)+f(u)-f(v), \quad \forall u, v \in D .
$$




\section{General nonlinear variational-like inequalities}

Since $T, A, N, a$, and $\eta$ are continuous and $f$ is lower semicontinuous, it follows that for each $v \in D$, the functional $u \mapsto \varphi(v, u)$ is weakly lower semicontinuous on $D$. We claim that $\varphi$ satisfies the condition (b) of Lemma 2.5. If it is false, there exist a finite set $\left\{v_{1}, \ldots, v_{m}\right\} \subset D$ and $u=\sum_{i=1}^{m} \lambda_{i} v_{i}$ with $\lambda_{i} \geq 0$ and $\sum_{i=1}^{m} \lambda_{i}=1$ such that $\varphi\left(v_{i}, u\right)>0$ for all $i=1, \ldots, m$, that is,

$$
\left\langle z^{*}-N(T u, A u), \eta\left(v_{i}, u\right)\right\rangle-a\left(u, v_{i}\right)+f(u)-f\left(v_{i}\right)>0
$$

for all $i=1, \ldots, m$. It follows that

$$
\sum_{i=1}^{m} \lambda_{i}\left\langle z^{*}-N(T u, A u), \eta\left(v_{i}, u\right)\right\rangle>\sum_{i=1}^{m} \lambda_{i} a\left(u, v_{i}\right)-f(u)+\sum_{i=1}^{m} \lambda_{i} f\left(v_{i}\right) \geq 0,
$$

which contradicts the condition that $N$ and $\eta$ have the 0 -diagonally concave relation with respect to $z^{*}$. Therefore, the condition (b) of Lemma 2.5 holds. Since $f$ is proper convex lower semicontinuous, it follows from [5] that its subdifferential $\partial f(v) \neq \varnothing$ for all $v \in$ $\operatorname{int}(\operatorname{dom} f)$. It is easy to see that $f(u) \geq f\left(v^{*}\right)+\left\langle r, u-v^{*}\right\rangle$ for all $v^{*} \in \operatorname{int}(\operatorname{dom} f) \cap D$, $r \in \partial f\left(v^{*}\right)$, and $u \in B$. For any fixed $v^{*} \in \operatorname{int}(\operatorname{dom} f) \cap D$, set

$$
Q=(\alpha+c)^{-1}\left[\delta\left(\left\|N\left(T v^{*}, A v^{*}\right)\right\|+\left\|z^{*}\right\|\right)+d\left\|v^{*}\right\|+\|r\|\right]
$$

and $K=\left\{u \in D:\left\|u-v^{*}\right\| \leq Q\right\}$. Then $K$ and $D_{0}=\left\{v^{*}\right\}$ are both weakly compact convex subsets of $D$. It follows that for each $u \in D-K$,

$$
\begin{aligned}
\varphi\left(v^{*}, u\right)= & \left\langle z^{*}-N(T u, A u), \eta\left(v^{*}, u\right)\right\rangle-a\left(u, v^{*}-u\right)+f(u)-f\left(v^{*}\right) \\
\geq & \left\langle z^{*}, \eta\left(v^{*}, u\right)\right\rangle+\left\langle N(T u, A u)-N\left(T v^{*}, A u\right), \eta\left(u, v^{*}\right)\right\rangle \\
& +\left\langle N\left(T v^{*}, A u\right)-N\left(F v^{*}, G v^{*}\right), \eta\left(u, v^{*}\right)\right\rangle \\
& -\left\langle N\left(F v^{*}, G v^{*}\right), \eta\left(v^{*}, u\right)\right\rangle \\
& +a\left(v^{*}-u, v^{*}-u\right)-a\left(v^{*}, v^{*}-u\right)+\left\langle r, u-v^{*}\right\rangle \\
\geq & \left\|u-v^{*}\right\|\left[(\alpha+c)\left\|u-v^{*}\right\|-\delta\left(\left\|N\left(T v^{*}, A v^{*}\right)\right\|+\left\|z^{*}\right\|\right)-d\left\|v^{*}\right\|-\|r\|\right]>0,
\end{aligned}
$$

that is, the condition (c) of Lemma 2.5 holds. By Lemma 2.5, there exists $\hat{u} \in D$ such that $\varphi(v, \widehat{u}) \leq 0$ for all $v \in D$, that is,

$$
\left\langle N(T \hat{u}, A \hat{u})-z^{*}, \eta(v, \hat{u})\right\rangle+a(\hat{u}, v-\widehat{u}) \geq f(\widehat{u})-f(v), \quad \forall v \in D .
$$

Now we prove that $\hat{u}$ is a unique solution of the general nonlinear variational-like inequality (2.1). Suppose that $u_{1}$ and $u_{2}$ are two solutions of the general nonlinear variational-like inequality (2.1). It follows that

$$
\begin{aligned}
& \left\langle N\left(F u_{1}, G u_{1}\right)-z^{*}, \eta\left(u_{2}, u_{1}\right)\right\rangle+a\left(u_{1}, u_{2}-u_{1}\right) \geq f\left(u_{1}\right)-f\left(u_{2}\right), \\
& \left\langle N\left(F u_{2}, G u_{2}\right)-z^{*}, \eta\left(u_{1}, u_{2}\right)\right\rangle+a\left(u_{2}, u_{1}-u_{2}\right) \geq f\left(u_{2}\right)-f\left(u_{1}\right) .
\end{aligned}
$$


Using $\eta(u, v)=-\eta(v, u)$ for all $u$ and $v \in D,(3.7)$, we deduce that

$$
\begin{aligned}
0 & \geq\left\langle N\left(T u_{1}, A u_{1}\right)-N\left(T u_{2}, A u_{2}\right), \eta\left(u_{1}, u_{2}\right)\right\rangle+a\left(u_{1}-u_{2}, u_{1}-u_{2}\right) \\
& \geq(\alpha+c)\left\|u_{1}-u_{2}\right\|^{2} \geq 0,
\end{aligned}
$$

which means that $u_{1}=u_{2}$ and $\hat{u}$ is the unique solution of the general nonlinear variational-like inequality (2.1). This completes the proof.

Theorem 3.2. Let $D, B, B^{*}, \eta, a, f, T$, and $N$ be as in Theorem 3.1, let $A: D \rightarrow B^{*}$ be Lipschitz continuous with constant $\gamma$, and let $N: B^{*} \times B^{*} \rightarrow B^{*}$ be $\eta$-relaxed monotone with constant $\alpha$ with respect to $T$ in the first argument and Lipschitz continuous with constant $\beta$ in the second argument. Assume that $N$ and $\eta$ have the 0 -diagonally concave relation with respect to $z^{*} \in B^{*}$ and $c>\alpha+\beta \gamma \delta$. Then the general nonlinear variational-like inequality (2.1) has a unique solution $\hat{u} \in D$.

Proof. Let

$$
Q=(c-\alpha-\beta \gamma \delta)^{-1}\left[\delta\left(\left\|N\left(T v^{*}, A v^{*}\right)\right\|+\left\|z^{*}\right\|\right)+d\left\|v^{*}\right\|+\|r\|\right]
$$

and $K=\left\{u \in D:\left\|u-v^{*}\right\| \leq Q\right\}$. It follows from the proof of Theorem 3.1 that

$$
\begin{aligned}
\varphi\left(v^{*}, u\right)= & \left\langle z^{*}, \eta\left(v^{*}, u\right)\right\rangle+\left\langle N(T u, A u)-N\left(T v^{*}, A u\right), \eta\left(u, v^{*}\right)\right\rangle \\
& -\left\langle N\left(T v^{*}, A u\right)-N\left(T v^{*}, A v^{*}\right), \eta\left(v^{*}, u\right)\right\rangle \\
& -\left\langle N\left(T v^{*}, A v^{*}\right), \eta\left(v^{*}, u\right)\right\rangle \\
& +a\left(v^{*}-u, v^{*}-u\right)-a\left(v^{*}, v^{*}-u\right)+\left\langle r, u-v^{*}\right\rangle \\
\geq & \left\|u-v^{*}\right\|\left[(c-\alpha-\beta \gamma \delta)\left\|u-v^{*}\right\|\right. \\
& \left.\quad-\delta\left(\left\|N\left(T v^{*}, A v^{*}\right)\right\|+\left\|z^{*}\right\|\right)-d\left\|v^{*}\right\|-\|r\|\right]>0 .
\end{aligned}
$$

The rest of the proof follows precisely as in the proof of Theorem 3.1. This completes the proof.

Remark 3.3. Theorems 3.1 and 3.2 improve Ding's [1, Theorems 3.1 and 3.2] and Yao's [10, Theorem 3.1].

\section{Acknowledgments}

The authors would like to thank the referee for a critical reading of the manuscript and many valuable suggestions. This work was supported by the Science Research Foundation of Educational Department of Liaoning Province (2004C063) and Korea Research Foundation Grant (KRF-2003-005-C00013).

\section{References}

[1] X. P. Ding, Algorithm of solutions for mixed-nonlinear variational-like inequalities in reflexive Banach space, Appl. Math. Mech. 19 (1998), no. 6, 521-529.

[2] General algorithm for nonlinear variational-like inequalities in reflexive Banach spaces, Indian J. Pure Appl. Math. 29 (1998), no. 2, 109-120. 


\section{General nonlinear variational-like inequalities}

[3] __ Existence and algorithm of solutions for nonlinear mixed variational-like inequalities in Banach spaces, J. Comput. Appl. Math. 157 (2003), no. 2, 419-434.

[4] X. P. Ding and K.-K. Tan, A minimax inequality with applications to existence of equilibrium point and fixed point theorems, Colloq. Math. 63 (1992), no. 2, 233-247.

[5] I. Ekeland and R. Temam, Convex Analysis and Variational Problems, Studies in Mathematics and Its Applications, vol. 1, North-Holland, Amsterdam, 1976.

[6] Z. Liu, S. M. Kang, and J. S. Ume, Completely generalized multivalued strongly quasivariational inequalities, Publ. Math. Debrecen 62 (2003), no. 1-2, 187-204.

[7] The solvability of a class of quasivariational inequalities, Adv. Nonlinear Var. Inequal. 6 (2003), no. 2, 69-78.

[8] Z. Liu, J. S. Ume, and S. M. Kang, Nonlinear variational inequalities on reflexive Banach spaces and topological vector spaces, Int. J. Math. Math. Sci. 2003 (2003), no. 4, 199-207.

[9] Completely generalized quasivariational inequalities, Adv. Nonlinear Var. Inequal. 7 (2004), no. 1, 35-46.

[10] J. C. Yao, Existence of generalized variational inequalities, Oper. Res. Lett. 15 (1994), no. 1, 3540.

Zeqing Liu: Department of Mathematics, Liaoning Normal University, P.O. Box 200, Dalian, Liaoning 116029, China

E-mail address: zeqingliu@dl.cn

Juhe Sun: Department of Mathematics, Liaoning Normal University, P.O. Box 200, Dalian, Liaoning 116029, China

E-mail address: juhesun@163.com

Soo Hak Shim: Department of Mathematics and Research Institute of Natural Science, Gyeongsang National University, Jinju 660-701, Korea

E-mail address: math@nongae.gsnu.ac.kr

Shin Min Kang: Department of Mathematics and Research Institute of Natural Science, Gyeongsang National University, Jinju 660-701, Korea

E-mail address: smkang@nongae.gsnu.ac.kr 


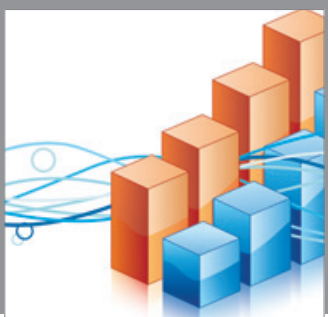

Advances in

Operations Research

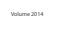

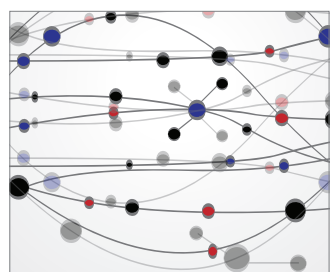

\section{The Scientific} World Journal
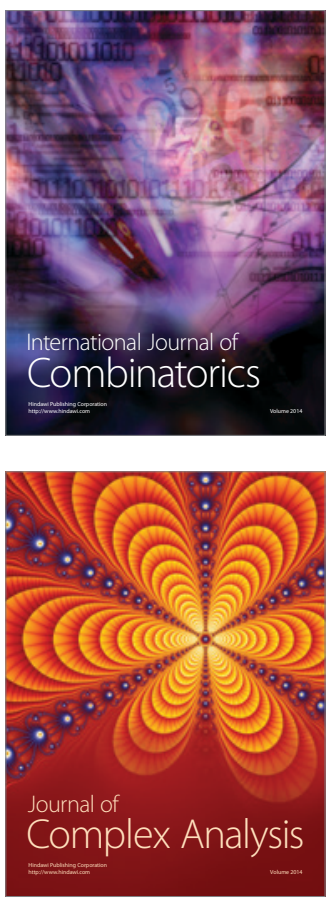

International Journal of

Mathematics and

Mathematical

Sciences
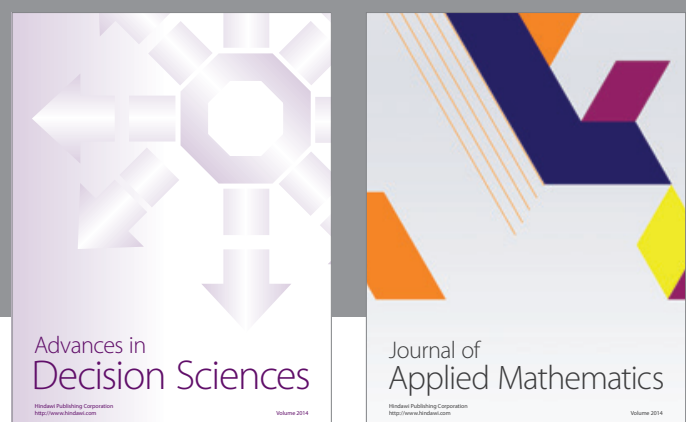

Journal of

Applied Mathematics
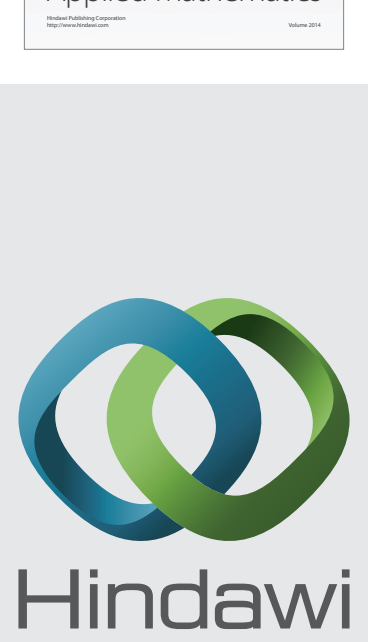

Submit your manuscripts at http://www.hindawi.com
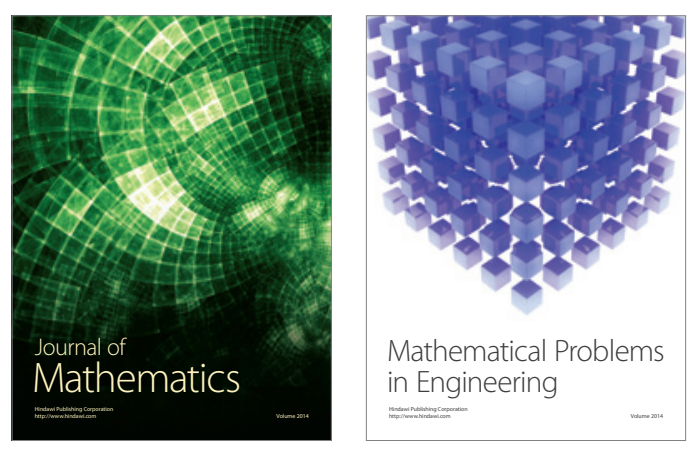

Mathematical Problems in Engineering
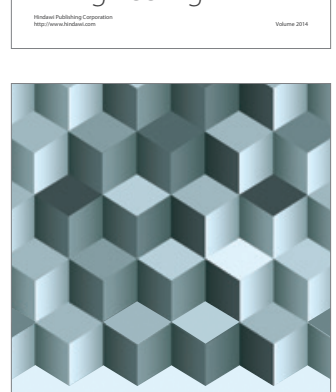

Journal of

Function Spaces
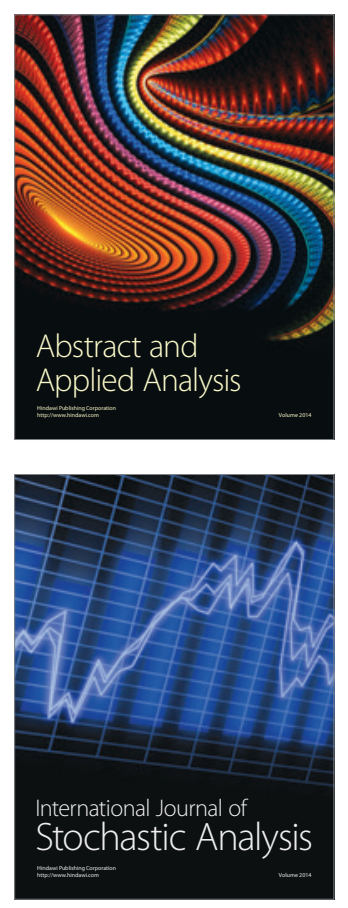

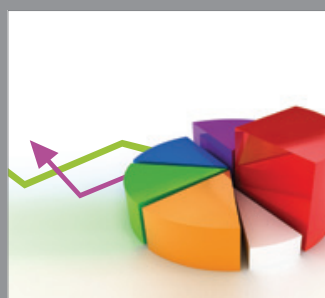

ournal of

Probability and Statistics

Promensencen
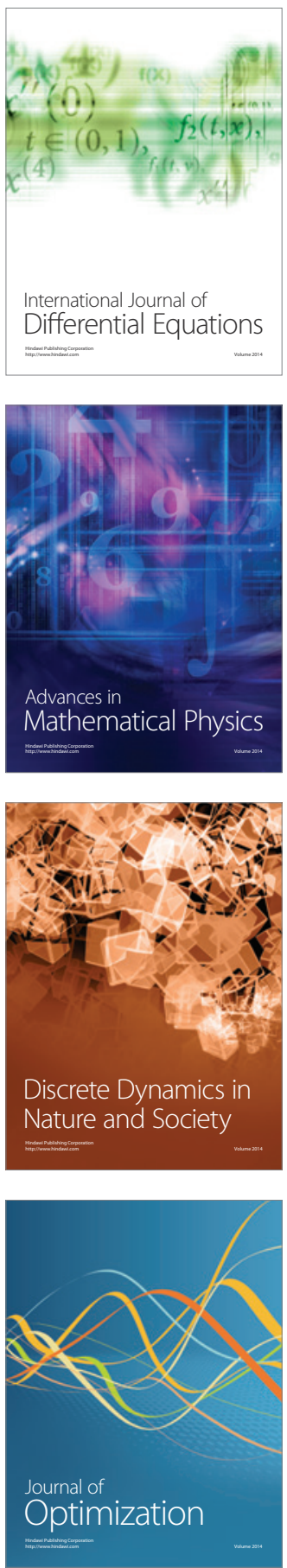